\title{
2 Theoretical Perspectives on Adolescents in the Family
}

In this chapter our goal is to focus on theories of adolescence and adolescent development. Theories have two basic functions: the first is to organize and give meaning to what we understand about an area and the second is to guide researchers by suggesting hypotheses that they can test in their experiments (Goossens, 2006). For example, attachment theory, which we will explore later, gives researchers ideas about how those adolescents who are secure and those who are insecure in terms of their attachment to parents are likely to behave in particular situations, such as in relating to their mothers (Becker-Stoll, Fremmer-Bombik, Wartner, Zimmerman \& Grossman, 2008) or becoming involved with a romantic partner (Hazan \& Zeifman, 1994).

As previously mentioned, in early theories of adolescence, this phase of development was seen as a period of storm and stress, because of puberty (Freud, 1958) and the strong mechanisms of defence needed to control sexual impulses at this time of life, or because of changes in the emotional life of adolescents (Hall, 1904). According to Hall, because the higher emotions (e.g., sympathy, love, aesthetic enjoyment) are only developing at this stage, adolescents are subject to continuous oscillations between emotional extremes. Examples of such extremes or oppositions include sensitiveness and dullness, self-confidence and humility, selfishness and altruism, wisdom and folly.

According to the 'storm and stress' theory, adolescents were expected to experience (and display) frequent mood swings, to have difficult relationships with their parents, and to be prone to risk-taking (Arnett, 1999). While these characteristics may sound familiar to those who live or work with adolescents, particularly troubled adolescents, it is problematic to assume that all adolescents everywhere experience these problems to a similar degree. We now know that most adolescents survive this period of their lives without extreme mood swings, have relatively harmonious relationships with their parents and don't take risks like driving at high speed while under the influence of alcohol (Arnett, 1999).

Nevertheless, adolescence is generally perceived, especially in Western cultures, as a difficult time for many young people because during this stage, they need to establish their own unique identity and also deal with pressure from their peer group and respond to the expectations of society (Northrup \& Bean, 2007). Hill and her colleagues argue that adolescence "is marked by change and renegotiation in almost every arena” (p.367; Hill, Bromell, Tyson \& Flint, 2007). Relationships with parents need to be renegotiated to reflect the greater maturity and needs for autonomy of the adolescent. In addition, biological, social and cognitive development are likely to result in big changes in all three areas. 
The anthropologist, Margaret Mead (1928), studied young people on an island in Samoa who had little contact with the west and showed that the 'storm and stress' experience was not universal but largely determined by the broader culture in which the young people lived. Of course, it is arguable whether finding one very different culture where such a phenomenon does not appear to exist really 'proves' that the storm and stress experience is not common among adolescents.

Other researchers such as Daniel Offer (e.g., Offer, Ostrov \& Howard, 1982) and Reed Larson (Larson \& Richards, 1994; Larson, Richards, Moneta, Holmbeck \& Duckett, 1996) have found more concrete evidence that most adolescents cope effectively with this stage of their lives. It is important to note that these studies were carried out with normal community adolescents and are not based on the experiences of the "neurotic Viennese women" with whom Freud worked, or even on adolescents who could be labelled as having mental health problems.

\subsection{Family Systems Theory}

From a systems theory perspective, the family is seen as "a dynamic whole composed of constantly shifting inter-relationships but still bounded and rule-governed" (Sieburg, 1985, p.xi). In other words, the focus is not so much on the individual members of the family, but on how the behaviour of each family member affects the others. The impact that each member has on other members, and on the system as a whole, is seen as systematic and predictable (Cox \& Paley, 1997; Parke, 2004a).

According to Family Systems theory, there are also subsystems such as the parental subsystem (which may involve two resident parents, one resident and one non-resident parent, one resident biological parent and a step-parent, and a non-resident parent, or a single parent) and the child subsystem (involving all the children in the family). In a step- or blended family, the child subsystem might be divided between his children and her children, or even his children, her children and their children.

The basic principles of Family Systems Theory include:

- the whole family system is greater than the individual contributions of members;

- change in one member of the family will affect other members and the system as a whole;

- families can react to the same experience quite differently and similar experiences do not necessarily lead to the same outcome;

- families need to be able to change in response to both factors internal to the family and to external factors such as changes in society;

- family members tend to work together to restore the status quo following a difficult event;

- behaviour is primarily communicative, with the most important communication being about the relationships between individuals;

- Families have rules, both implicit and explicit, that define the nature of the family. 
Family Systems Theory (FST) changed the way scholars think about families in several ways. The focus moved from individual members to the family as a whole, especially in two important areas: family breakdown and the way therapy is approached (Noller \& Fitzpatrick, 1993). For example, "no fault divorce" can be seen as an outcome of FST, with the focus on the system rather than on the bad behaviour of any individual. Family therapy could also be seen as an outcome of FST, because the focus is on the complex interactions among family members, rather than on the "identified patient" who might be a scapegoat for the rest of the family members.

From an FST perspective, the rules governing the system need to be flexible and negotiable in response to the changing needs of the developing adolescent. Changes in parents' behaviour and the family climate can be predicted by the attributes and behaviours of adolescents. Further, the family environment is a product of the input of both the parent/s and the adolescents (Persson, Stattin \& Kerr, 2004; Stattin, Persson, Burk \& Kerr, 2011). Stattin et al. argue that democratic family systems are created by the interdependence of parent/s and adolescents, with mutual openness and responsiveness being important. These authors found that both adolescent and parent behaviour predicted changes in adolescents' perceptions of the family climate. In addition, when changes occurred, the behaviour of both parent and adolescent were affected.

Family Systems Theory plays an important role in our understanding of families and the individuals within them. For example, a crucial change that has occurred because of FST is the emphasis on family therapy, with the identified patient seen as a product of the family system rather than a problem in his or her own right. Family therapy focuses on "fixing" the family system, rather than focusing on the individual, who may just revert to the problematic behaviour when returned to the family following therapy.

\subsection{Cognitive Theories}

Cognitive theorists such as Piaget (Inhelder \& Piaget, 1958) and Elkind (1967) focused their attention on the changes in thinking that occur during adolescence. Adolescents become capable of formal operations, meaning that their thinking becomes more abstract and they can consider various possibilities, deal with propositions and plan ahead. These features should mean that parents and adolescents are able to engage in meaningful conversations about issues that affect the adolescent's future such as study and travel plans. The research that we mentioned in Chapter 1 about the development of the adolescent brain, however, would suggest that adolescents need help from their parents in working through decisions.

According to Piaget and his colleagues, adolescents tend to be idealistic, but in a naïve kind of way, thinking that they can change the world for the better. He argues that an important step towards adulthood is to become more realistic. On the 
other hand, a more positive approach may be to harness this idealism in ways that benefit society. For example, in the recent (2011) disastrous floods in Queensland, Australia, many young people were among the army of volunteers who went out helping complete strangers whose homes had been flooded to clean the mud out of their houses and clean up possessions that could be salvaged. Some of these young volunteers came from interstate and even from as far away as Texas, USA. Family influences such as belonging to a family that is highly community-minded or that has a focus on caring for those in need are likely to play a large part in a young person's willingness to be a volunteer in such circumstances.

\subsubsection{Adolescent Egocentrism}

Another cognitive theory about adolescent behaviour is Elkind's (1967) focus on what he calls the egocentrism of adolescents who are likely to be very preoccupied with themselves and their own issues and tend to think that others are preoccupied with them as well. From this preoccupation comes the self-consciousness that is so much a part of the adolescent experience. Elkind describes the egocentrism of adolescence as "the failure to differentiate between the cognitive concerns of others and those of the self" (p.1025). He also describes how adolescents tend to see themselves as surrounded by an imaginary audience and how they construct a personal fable for themselves. One of the best examples of the imaginary audience in recent literature is in Ian McEwan's “Atonement”, where the 13 year-old Briony imagines her mother's funeral with herself as the complete focus of attention (see Box). According to Goossens (2006a), this concept of the imaginary audience may also help to explain why adolescents are so self-conscious, because they project both their good and bad feelings onto their imaginary audience, an audience that they believe is always watching.

\footnotetext{
"Her mother was forty-six, dispiritingly old. One day she would die. There would be a funeral in the village at which Briony's dignified reticence would hint at the vastness of her sorrow. As her friends came up to murmur their condolences they would feel awed by the scale of her tragedy. She saw herself standing alone in a great arena, within a towering colosseum, watched not only by all the people she knew but by all those she would ever know, the whole cast of her life assembled before her in her loss. And at the churchyard, in what they called the grandparent's corner, she and Leon and Cecilia would stand in an interminable embrace in the long grass by the new headstone, again watched. It had to be witnessed. It was the pity of these well-wishers that pricked her eyes."
}

McEwan, L (2003)

Box 2.1: Adolescent Imaginary Audience 
The concept of personal fable refers to the adolescent belief about being special and unique (in the sense of better than others) and invulnerable to harm, or invincible. For example, they may believe that smoking will not harm them (Frankenberger, 2004), that they can drive a car without passing the test, or have unprotected sex or engage in binge-drinking without the consequences that might result for other people (Schwartz, Maynard \& Uzelac, 2008). While these concepts are interesting and provide for fascinating anecdotes, there does not seem to be extensive research into these phenomena or the association between these phenomena and family processes. In addition, as we noted in Chapter 1, there is evidence that although adolescents understand the dangers of risky behaviour, they are likely to engage in these behaviours, to varying degrees, in any case (Steinberg, 2007).

\subsection{Individuation}

There are two somewhat related processes that adolescents need to go through in order to become mature adults: separation-individuation and establishing an independent identity. Although these two processes of individuation and identityformation are discussed separately, they are clearly related and may be occurring simultaneously. In a sense, engaging in the separation-individuation process makes it easier to develop a separate identity and developing a separate identity makes the individuation process easier. Erikson (1968) saw identity formation as the central outcome of individuation.

Separation-individuation, as the name suggests, can be seen as two separate but related processes (Bray, Adams, Getz \& McQueen, 2003; Silverberg \& Gondoli, 1996). According to Bray et al., individuation reflects an individual's need for close intimate relationships with parents or parental figures while still being able to operate autonomously and make many of one's own decisions. Separation, on the other hand, can involve an unhealthy detachment from the family and a rejection of parental values in favour of those of peers. More recent discussions have focused on whether it is more helpful to define individuation in terms of autonomy or independence (e.g., Van Petegem, Vansteenkiste, \& Beyers, 2013).

The process of individuation does not necessarily involve leaving the family home or breaking ties with family members but is likely to involve a gradual change (probably not fast enough for adolescents) in which they have more influence on decisions involving themselves, and parents have less influence. As Crespi and Sabatelli (1997) note, young people at this stage are expected to rework their ties to their family members and to establish "a separate yet connected self" and come to see themselves as separate and distinct within their social network of family and friends. They also need to renegotiate their relationships with their parents so that they are no longer a child being 'controlled' by a parent but a person of more equal status seeking relationships of greater mutuality with their parents. The task for adolescents is to 
develop increasing autonomy while, at the same time, maintaining a strong sense of connection and a meaningful emotional bond with their parents (PalladinoSchultheiss, \& Blustein, 1994).

In their classic study of individuation in adolescents, Grotevant and Cooper (1986) analysed the family interactions of adolescents. They focused on two qualities: individuality and connectedness because they believed that these two factors were important for the development of healthy adolescents. Individuality was important because it allowed the adolescents to see themselves as distinct from other family members and thus to both express their own views and acknowledge the views of other family members who were separate from them. Their connectedness to other family members, on the other hand, gave them the security to express their views without the fear of being "shot down in flames".

As the work by Grotevant and Cooper (1986) shows, this important process of individuation can be either helped or hindered by parental reactions to adolescents' attempts at individuation. Cobb (1998) argues that how easy or difficult adolescents find this transition depends on the kind of support they receive, particularly from their parents. Where the adolescents are able to experience real connectedness to other family members and are secure in their presence, real sharing of ideas could take place. In an Australian study, Noller and Callan (1986) used the two main dimensions of Olson's Circumplex model (Olson et al., 1983) and found that adolescents wanted high levels of adaptability in their families so they could make decisions for themselves about what they would do, but they also wanted high levels of cohesion or closeness so that they could be confident in the support of their parents. Vansteenkiste and colleagues (Vansteenkiste, Soenens, Duriez \& Van Petegem, 2014) found that whether adolescents internalised rules or became defiant depended on the way that the rule was introduced. The degree of rule enforcement was not as important as the way rules were communicated.

Because individuation develops more easily when family relationships are healthy, this process can be seen as a "co-construction by children and their parents" (Reis \& Buhl, 2008, p.369). The role of parents is to encourage individual adolescents to think, feel and act independently of other family members and the process can be explained in terms of parent-child transactions during this family and life stage (Koepke \& Denissen, 2012). Where this kind of differentiation is not encouraged, family members' individuality is likely to be negated. A worst-case scenario is when having different ideas is seen as disloyalty and a threat to the stability of the family (Crespi \& Sabatelli, 1997). Those who struggle to individuate are likely to become overdependent on family members and over-identified with them. Paradoxically, those who successfully individuate tend to have a greater capacity for close relationships without the risk of being overly dependent on them.

Even in divorced or separated families, the quality of relationships with both the mother and the father is related to the separation-individuation process (Jones, Kramer, Armitage, \& Williams, 2003). Young adults whose parents were divorced by 
the time they were adolescents indicated much higher levels of blurred boundaries within their families during their adolescence (Perrin, Ehrenberg \& Hunter, 2013).

Jones and colleagues found that boys whose fathers lived elsewhere were more separated from their fathers on some dimensions, although this difference was less clear when the boys had a high quality relationship with both of their parents. The more contact the son had with his non-resident father, the more likely he was to achieve a healthy separation, although he was still likely to maintain strong dependence on his father. (See Chapter 5 for more information on adolescents in divorced or separated families).

Although there are not a lot of differences between males and females in terms of the processes involved in separation-individuation, there is evidence that males tend to shun dependent ties, whereas females tend to have a greater capacity for forming close relationships with their peers (Gnaulati \& Heine, 2001). These findings would seem to provide support for the view that modern males eschew commitment and are less willing to marry or be involved in committed relationships.

Comparisons of the individuation process between adolescents in the USA and in European countries showed that the relative power of adolescents was greater in German families than in American families (Laursen, Wilder, Noack \& Williams, 2000; Noack \& Buhl, 2004). This finding may be a function of a higher focus on authority in the USA (Reis \& Buhl, 2008). German adolescents reported being closer to their mothers than did American adolescents, although there were no differences with regard to closeness to fathers (Laursen et al., 2000). It is important to remember, however, that individuation is likely to be different in Western countries where there is a much stronger focus on individualism and agency (having the power to take action) than is true for collectivist cultures such as Turkey (Kagitcibasi, 2005).

One problem for Western societies in particular is that too much emphasis on individuation can lead to the kind of rampant individualism that we see in the media where an individual is supported by the media in believing that he or she is 'the most important person in the world' and should do what is best for him or herself even if others could be hurt by such selfish behaviour. Collectivist cultures tend to put more emphasis on what is good for the family or the society rather than on what is good for the individual. While such an approach may sometimes 'cramp the style' of individuals, not allowing them the freedom that is available in the West, it may also produce a more harmonious society. Collectivist countries can be found in Asia, Africa, the Middle East and the Pacific Islands.

Psychiatrist Stuart Hauser and his colleagues who carried out a 30-year longitudinal study known as the Adolescent Families Development Project (see Allen, 2010; Hauser \& Allen, 2006; Hauser, Allen \& Golden, 2006) were particularly interested in exploring the ways in which family interactions during adolescence impact on adolescents' psychosocial development. The sample for this study involved 146 young people, all of whom were 14 years of age. Half the sample were Boston High School students and the other half were psychiatric patients who had been in hospital 
for a range of psychiatric disorders, although adolescents who suffered from thought disorder, mental retardation or organic disorders were excluded. The adolescents in the sample, who had also experienced serious abuse by close family members, lived for between two months and a year in the hospital.

Data collection was very comprehensive and included questionnaires and tests, open-ended interviews, and audio-taped observational data of parents and teens discussing disagreements. Working from an understanding that "a central task of adolescent development is the adolescent's need to establish autonomy while maintaining relationships with parents" (Allen, 2010, p. 243), Hauser, Allen and colleagues focused on the ways that adolescents' interactions with parents facilitated or hindered their individuation (Hauser, Borman, Jacobson, Powers, 1991; Hauser, Powers \& Noam, 1991). These researchers were interested in how adolescents reacted to parents' continual attempts to constrain their needs for more autonomy and the consequences of that type of parenting over time.

Hauser, Allen and colleagues focused on the expression of hostility and found that there was a modelling effect, with hostile behaviour on the part of parents producing hostile behaviour in adolescents. They also found that where adolescents struggled to achieve an appropriate level of autonomy, they tended to be more negative in their interactions over time (Allen, Hauser, Eickholt, Bell \& O’Connor, 1994) and more hostile to other family members (Allen, Hauser, O’Connor, Bell \& Eickholt, 1996).

A further consequence of parents constraining an adolescent's moves towards greater autonomy was greater hostility in their relationships with their peers. Adolescents whose strivings towards autonomy were undermined by their parents were more hostile than other adolescents in their relationships with their peers as many as ten years later (Allen, Hauser, O’Connor \& Bell, 2002). As Allen (2010) summarizes this aspect of the research, "frustration of a central developmental task was associated with more dysfunctional behaviour much later in development and in new contexts” (p.247).

\subsection{Identity Formation}

Every adolescent tends to reach a point in time when they realise that they can no longer continue behaving as a child and they have to put away childish things (Cobb, 1998). Adolescents face many changes and need to make sense of a range of new experiences and new expectations, and to forge a stable identity. Achieving a stable identity involves making a number of decisions about who one is and what one wants to become. As Kroger explains, the goal of adolescence is "To become faithful and committed to some ideological world view, ...to find a cause worthy of one's vocational energies and reflecting one's basic values" (Kroger, 2004, p.29). Kroger sees the establishment of a stable identity as "one of life's critical crossroads in the transition to adult life". 
Identity refers to a person's "stable, coherent and integrated sense of self" (Perosa, Perosa \& Tam, 1996, p.818). In other words, those with stable identities know who they are and what they stand for. A stable identity will involve a range of choices other than a vocational choice, including choices about political preferences, religious commitments and sexual orientation to name a few relevant areas. These choices are likely to be affected by family relationships whether, in extreme cases, an adolescent decides to adopt family values in their entirety or to rebel totally against those values and live on the street, for example (Pears \& Noller, 1995).

In earlier times, at least in Europe and the United Kingdom, identity tended to be restricted by social status and family ties that determined a person's position for life (Baumeister, 1987). For example, in the fairly rigid social class structure that permeated Britain in earlier times, individuals were expected to work within the confines of the social class of their family of origin (Jane Austen's "Pride and Prejudice" is about this issue). Young males would be expected to be a labourer or a tradesman or a professional in line with the work of their fathers. Boys would also be expected to attend a similar school to their father and to marry someone of their own class.

Forming an identity has come to be seen as a task that every adolescent needs to accomplish (Erikson, 1968). Part of the process of identity formation involves reflecting on the rules, roles and values that were adopted as a child in the family. Adolescents need to make decisions about which ones to continue and which to discard in the light of their own developing interests and values and their understanding of their own talents (Kroger, 2004). Erikson $(1968,1980)$ saw individuals as falling along a dimension from identity synthesis (those who have successfully worked through the process discussed above) and identity confusion. Those at the identity confusion end of the dimension are those who have difficulty developing a workable set of values and ideals that will be part of their adult identity (Schwartz, 2001).

Some writers have emphasized that adolescents are likely to be continually receiving feedback from the social environment, particularly from family and friends, as they explore alternative identities and commit to certain values and roles (Kerpelman \& Lamke, 1997). Some of this feedback will confirm them in the choices they have already made about identity, and some will lead them to think further about that identity. In addition, whereas some adolescents and young adults are likely to be open to such feedback even when it is not consistent with their own view of themselves, others are likely to seek only feedback that is in line with their own view and still others are likely to ignore all feedback. "It's my life and I'll do what I want".

Grotevant (1987) in his discussion of identity focused on the exploration process and noted that individuals are likely to differ in terms of the abilities and orientations that they bring to this process. For example, those who are rigid in their orientation and have few skills in critical thinking or problem-solving would have difficulty engaging in the kind of exploration of alternative possibilities needed to reach the stage of identity achievement. Meeus (2011), in his extensive review of longitudinal research on adolescent 
identity formation, argued that there is a lack of empirical research showing that young people actually engage in exploration before committing to an identity. He argued that family relationships are critical to this whole process of identity achievement. Meeus showed that for adolescents, having a mature identity is related to the level of warmth in the family. Those with a mature identity tend to show high levels of psychological adjustment, be positive in terms of their personalities and do well at school.

\subsubsection{Family Structure and the Development of Identity}

Benson and Johnson (2009) explored the ways in which family structure during the adolescent years affects the age at which young people self-identify as adults. According to their findings, those in families with their two biological parents were less likely than those in single-parent and stepfamilies to see themselves as adults. Two-parent adoptive families were similar to the biological families. These researchers argue that the differences can be explained by the lack of economic resources available to nonnuclear families. The findings for stepfamilies, however, indicated that other factors such as the quality of relationships and the level of responsibility afforded the young person as well as the level of parental monitoring had an impact on the adolescent's subjective sense of having attained adulthood.

\subsubsection{Ethnicity and identity}

An important aspect of identity development concerns ethnic identity. This issue has probably become more important, given the heavy rates of migration, particularly to the western world and the current emphasis on multiculturalism. Ethnic identity involves understanding one's ethnic group and one's membership in that group even when one is likely to be a victim of racial or ethnic stigmatization (Hill et al., 2007; Hughes, Hughes, Rodriguez, Smith, Johnson et al., 2006). For example, those young people who have developed a strong ethnic identity as African American tend to be more socially competent and perform better academically than their peers whose ethnic identity is weaker; they are also less likely to become involved in delinquent or acting-out behaviour (Yasui, Dorham \& Dishion, 2004).

Identity development for these adolescents was influenced by both the home environment and their peers (Hill et al., 2007; Hughes et al., 2006). In fact, Hill et al. see identity formation for adolescents in Western cultures as mainly occurring outside the family in interaction with peers. African American adolescents need to be socialized about the history and the cultural beliefs of African Americans and also about the identification and handling of discrimination. They also need to carry out the kind of identity exploration that leads to positive mental health outcomes, despite the difficulties likely to be encountered (French, Seidman, Allen \& Aber, 2006). 
In a study of the ethnic identity of a sample of African-American adolescents, having a higher level of ethnic identity was positively related to parents' income and family cohesion as well as to their interpersonal functioning and their self-esteem (Street, Harris-Britt and Walker-Barnes, 2009). Ethnic identity was negatively related to their reports of conflict in the family and to their levels of depression. Those adolescents who reported having a positive ethnic identity as well as high levels of cohesion in their family also reported the highest levels of psychological adjustment, supporting the importance of adolescents establishing, not only a stable and coherent identity, but also the need for that identity to be positive if they are to be well-adjusted.

Northrup and Bean (2007) discuss the situation of multiracial adolescents (That is where mother and father are of different ethnic backgrounds.) They focus on adolescents, one of whose parents is Latino (Hispanic) and the other is AngloAmerican. They see adolescents with a multiracial parentage as likely to have a particularly difficult time in establishing an identity. These authors argue that these young people "must develop this new identity and decide how, or even if, they can reflect positive aspects of all heritages while simultaneously rejecting social expectations and stereotypes” (p. 252).

Northrup and Bean (2007) focus on the special problems of adolescents who have at least one Latino parent, arguing that those whose Latino parent is white are likely to have an easier experience of identity formation and general social experience than those whose Latino parent is dark-skinned (Alvarez, 1998; Bonilla-Silva, 2004). In addition, youth with mixed-race parentage may not always be accepted by the extended families and racial groups into which they have been born (Deters, 1997). If one group is more accepting than the other group, a young person may decide to adopt that identity exclusively (Northrup \& Bean).

According to Hill et al. (2007) the development of ethnic identity often begins with a crisis such as experiencing discrimination or finding oneself in a more diverse group of peers in middle school or high school. Discrimination is obviously more likely to occur in groups involving young people from several different ethnic groups. Unfortunately, low-income African Americans are more likely than those of other ethnicities to experience discrimination. It is important to remember, however, that low-income Anglo-Americans are also likely to experience discrimination, particularly in schools involving whites from different SES groups.

\subsubsection{Marcia's identity statuses}

Based on his Identity Status Interview, Marcia (1966) defined four identity statuses that may characterize adolescents. Whereas some critics claim that Marcia's model is out-dated, Meeus (2011) claims that his work is the "most important elaboration" of Erikson's work (p.75) ever carried out. In addition, Schwartz (2001) has commented on the usefulness and versatility of the identity status model. Marcia's model was based 
on two dimensions. The first dimension concerns whether or not an individual has made a commitment to particular social roles and values, and the second concerns whether or not the commitment was preceded by a period of exploration. According to Marcia, the best commitments are made after a period of exploration that leads to a time of crisis and decision-making. (See Table 2.1)

Table 2.1: Marcia's Identity Statuses

\begin{tabular}{lll}
\hline & Period of Exploration & \\
\hline Commitment & Yes & No \\
Yes & Identity achieved & Foreclosure \\
No & Moratorium & Identity diffusion \\
\hline
\end{tabular}

Marcia argued that the identity-achieved individuals have been through a period of exploration concerning who they are and what they want from life, whereas foreclosed individuals have committed themselves to an identity without going through such a period of exploration. These individuals, who are often females (Perosa et al., 1996), tend to adopt "roles and values of childhood identification figures". For example, they may choose a career by deciding to 'follow in their mother's or father's footsteps' (Kroger, 2004, p.37); they may also decide, at a later time following some personal experience with that type of work that it doesn't really suit them. Neither of the other two groups, Moratoriums or Diffusions have committed themselves to a particular set of roles and values, but Moratoriums are at least going through a process of exploration, whereas Diffusions are not really exploring the different possibilities that need to be considered in working towards a stable identity.

In his review of the research on these identity statuses, Marcia $(1980,1993)$ concluded that those in the Identity-achieved status were most likely to be welladjusted, followed by those in the Foreclosure status. Those in the two groups characterized by low levels of commitment, Moratorium and Diffusion, were the least likely to be well adjusted, with those in the Diffusion status being the least well-adjusted of the four groups. This group was very similar to those Erikson (1968) described as identity-confused. It is also interesting to note that those in the groups high on exploration (Identity-achieved and Moratorium) were high in openness (that is, able to deal with the unfamiliar) and high in intellectual curiosity whereas those in the Identity-achieved and Foreclosure groups scored higher on conscientiousness than those in the two groups that were low on commitment (Clancy \& Dollinger, 1993; Kroger \& Green, 1996; Soenens, Duriez, \& Goossens, 2005).

Some studies show links between identity and parenting (Luyckx, Soenens, Vansteenkiste, Goossens \& Berzonsky, 2007; Schwartz, Mason, Pantin \& Szapoczik, 2009). Warm and supportive relationships with parents, as opposed to controlling relationships, were related to more advanced identity formation. Reis and Youniss 
(2004) found that over time, those adolescents whose identity development was stable or maturing were more likely to have improved communication with their mothers than those whose identity development regressed.

Beyers and Goossens (2008) explored the short-term changes in identity formation in late adolescents, and their association with changes in their relationships with their parents. These researchers found that parenting was critical to the process of identity formation. A context of supportive parenting facilitates adolescents' identity formation. Specifically, parents can assist their adolescent by being accepting and responsive and can inhibit their adolescent's identity development if they are rejecting or overly controlling.

There is also evidence that being involved in activities in one's community, particularly in serving that community, aids in the development of identity (Pancer et al., 2007; Pratt et al., 2003; Taylor \& Pancer, 2007. Pancer et al. found that the Activists and Helpers in their study were significantly more advanced in terms of their identity development than those who were less active or not involved at all. (See Chapter 1 for more detail on this study)

\subsubsection{Identity styles}

Another way of looking at identity formation involves focusing on the styles that individuals use in constructing a stable identity, and thus focusing on the process rather than on identity status, given that personal identity tends to be constructed in social interactions with family, friends and others (Berzonsky, 1989, 1990). Combining the two approaches actually aids our understanding of the strong association between style and status (Schwartz, 2001). Berzonsky suggested three styles: informational, normative and diffuse-avoidant. (See Table 2.2)

Table 2.2: Identity Styles

\begin{tabular}{ll}
\hline Identity Style & Description \\
\hline Informational style & Seek out information \\
& Reflect and work on problem \\
& Flexible commitments \\
& Related to identity-achieved status \\
& Highly conforming \\
Noly on authority figures \\
Rigid and dogmatic commitment \\
Related to foreclosure status \\
Put off making decisions \\
React emotionally rather than rationally \\
Liffuse-avoidant style & Related to diffused status \\
\hline
\end{tabular}


The informational style involves having high self-esteem, seeking out information, working on the problem, making flexible commitments and being willing to reflect on the issue. This style is generally seen as the most adaptive style (Berzonsky, 1992; Schwartz, 2001). A person graduating from high school who carefully checks out potential courses and gets as much information about them as he can, keeping his options open until he has all the information, could be seen as operating in the informational style which is strongly related to the identity-achieved status (Berzonsky, 1993; Berzonsky \& Neimeyer, 1994). These adolescents are likely to have parents who use an authoritative style (see Chapter 3 on family environment for further discussion of parenting styles) and encourage exploration and negotiation in their youngsters.

The normative style involves a closed-minded approach rather than exploration (Berzonsky, 1993) and is associated with the Foreclosure status. Thus those with a normative style tend to be highly conforming, relying heavily on authority figures such as parents, and their commitment tends to be rigid and dogmatic in line with their highly stable identity (Berzonsky \& Neimeyer, 1994). As Schwartz (2001) notes, "the normative style involves passive copying of external standards and strong resistance toward any attempt to change those standards" (p.23). Someone with a normative style who was graduating from high school would be likely to take the course suggested by authority figures such as parents and teachers rather than do the kind of exploration that someone with an informational style would do. These adolescents are likely to have parents who use an authoritarian parenting style. In these families, obedience is likely to be a stronger value than encouraging adolescents to explore and negotiate and work things out for themselves.

Adolescents with a diffuse-avoidant style as the name suggests tend to put off making decisions and commitments and to deal with each situation as it comes, rather than having an integrated approach to life (Berzonsky, 1992). They are not good at problem solving and tend to react to situations emotionally rather than rationally. This style is associated with low levels of commitment, as well as low self-esteem and an unstable view of oneself. These adolescents have little concern about what the future holds for them or about the long-term consequences of the choices that they make. This style is most closely related to the diffused status in Marcia's model (Berzonsky, 1989; Schwartz, 1998). Adolescents with a diffuse-avoidant style are likely to come from families where the parents' style is permissive, leaving their youngsters to work things out for themselves without much guidance or negotiation.

\subsection{Attachment Theory}

Attachment theory is based on the assumption that a person's relationship with the mother (or primary carer) in infancy has a profound effect on that person's adjustment and future relationships (Bowlby, 1969, 1973, 1980). The responsiveness 
of the mother is seen as critical to whether the child develops secure or insecure attachment. If the mother is responsive when the child cries and is able to calm him, then the child is likely to be secure in attachment. Secure children are able to explore their environments and learn and grow, with confidence that their parents will be there for them when they are needed. According to Bowlby, on the basis of early family relationships, individuals develop working models about themselves, others and how relationships work. Those who develop positive working models about themselves and others are more likely to experience secure attachment and to have more satisfying relationships throughout their lives (Davila, 2011). Bowlby did not claim that these patterns are unchangeable, although they may be difficult to change.

\subsubsection{Attachment Functions}

Secure attachment relationships are seen as performing four functions: proximity seeking (the child wants to be near the attachment figure), separation protest (the child objects to separation from the attachment figure at least when very young), secure base (confidence in the availability of the attachment figure allows the child to explore the environment) and safe haven (when distressed the child can return to the attachment figure and expect comfort and protection).

Attachment security affects the quality of family relationships in adolescence. Securely-attached adolescents can balance autonomy and attachment needs more readily than insecure adolescents (Allen \& Hauser, 1996). Productive problem-solving approaches that both assert the adolescent's opinion and preserve relationships with parents tend to be a feature of discussions between parents and their securely-attached adolescents. In contrast, discussions between insecure adolescents and their parents tend to feature avoidance of problem-solving, anger and coercion. Interestingly, these difficulties could be predicted from attachment insecurity as assessed in infancy and at age six (Becker-Stoll \& Fremmer-Bombik, 1997).

Although adolescence is a stage where the young person is attempting to decrease their dependence on parental relationships, insecure adolescents are likely to find their striving for autonomy increases the impact of parental relationships on their lives. Allen and Land (1999) argue that families of insecure adolescents may have particular difficulties with the adolescent's attempts to renegotiate the relationship to achieve greater autonomy and respond with behaviours that reinforce the insecure attachment.

Although adolescents seek to establish an increased level of autonomy and independence, research indicates that this autonomy is best achieved in the context of secure relationships with parents (Allen, Hauser, Bell \& O'Connor, 1994; Allen \& Land, 1999, Fraley \& Davis, 1997). Adolescents who are securely attached are able to develop autonomy at the same time as relating well to parents, other family members and peers (Allen \& Hauser, 1996; Becker-Stoll, Fremmer-Bombik, Wartner, 
Zimmerman, \& Grossman, 2008). In fact, a critical developmental task for the adolescent is being able to function independently of parents in terms of the social, emotional and cognitive aspects of their lives, while at the same time maintaining a close relationship with them (Allen et al., 1994; Allen \& Land, 1999). The importance of a secure attachment relationship with parents is underlined by the finding that infant attachment security is associated with autonomy and closeness in adolescence (Becker-Stoll \& Fremmer-Bombik, 1997; Becker-Stoll et al., 2008). In other words, those who are secure in attachment are most likely to achieve a good balance between their own autonomy and their closeness with their parents.

Allen and his colleagues (Allen et al., 1994) were also able to use their longitudinal data referred to in Chapter 1 to understand the consequences of experiencing severe psychiatric disorders in early adolescence (by 14 years of age). They found that almost all of those hospitalized adolescents were insecure in attachment when they were 25 years of age. One of the ways that this insecurity was manifested was in the fact that they had not been able to resolve the traumatic events that had caused their hospitalization in the first place (Allen, Hauser, \& Borman-Spurrell, 1996). Insecurity of attachment was related to current (i.e., at age 25) involvement in criminality and the use of hard drugs, even when the researchers controlled for the fact that they had spent time in a psychiatric institution. Allen (2010) comments that insecurity of attachment seems to not only be a marker of psychiatric problems, but also is predictive of later problems.

There is evidence that secure adolescents are likely to have more positive experiences of romantic relationships than are insecure adolescents. Davila (2011) focuses on the impact of attachment security on romantic relationships in adolescence. Greater security is related to more positive expectations of marriage and future relationships and experiencing less depression in their adolescent romantic relationships. Further, secure adolescents are also less likely to stay in an unhappy relationship.

The literature is clear that the attachment security developed in the family has a direct impact on relationships both in adolescence and into the future. Laible (2007) found that "secure attachment relationships foster appropriate social behaviour by promoting high levels of emotional awareness, empathy, positive expressiveness, and low levels of negative dominant expressiveness"(p.1185).

\subsubsection{Attachment Styles}

Individuals can also be categorized into different attachment styles. Mary Ainsworth and her colleagues (Ainsworth, Blehar, Waters \& Wall, 1978) identified three major infant attachment styles based on the behaviour of the infants and the quality of care- giving of the primary carers. Hazan and Shaver (1987) used this typology to describe and assess attachment styles in adult romantic relationships, 
including those of adolescents. They found that the percentages of individuals endorsing the different styles were similar to those found for infants ( 56 percent claimed they were secure, 23 percent claimed they were avoidant and 25 percent claimed they were anxious-ambivalent). Those who claimed to be secure tended to describe their relationships with their parents as warm, avoidants described their mothers as cold and rejecting and anxious-ambivalents saw their fathers as unfair.

Bartholomew and Horowitz (1991), on the other hand, argued, based on Bowlbys' theorising, that attachment styles could be described in terms of attitudes to oneself and to others.

- Those who had positive views of both themselves and others were categorized as Secure. These individuals tended to be comfortable with both intimacy and autonomy.

- Those who had negative views of both themselves and others were categorized as Fearful. They tended to be uncomfortable getting close to others and to worry a great deal about being hurt.

- Those who had positive views of themselves and negative views of others were categorized as Dismissing. They tended to downplay the importance of relationships and put a lot of emphasis on being independent and selfsufficient.

- Those who had a negative view of themselves and a positive view of others were classified as Preoccupied, and tended to want very close relationships but also worried a lot about being hurt or abandoned. (See Table 2.3).

Table 2.3: Characteristics of Four Styles of Attachment

\begin{tabular}{|c|c|c|}
\hline \multicolumn{3}{|c|}{ MODEL OF SELF } \\
\hline MODEL OF OTHER & Positive & Negative \\
\hline Positive & $\begin{array}{l}\text { SECURE } \\
\text { Easy to get close to others } \\
\text { Comfortable depending on others } \\
\text { Comfortable with others depending on } \\
\text { them } \\
\text { Don't worry about being alone or not } \\
\text { accepted }\end{array}$ & $\begin{array}{l}\text { PREOCCUPIED } \\
\text { Want to be completely emotionally } \\
\text { intimate with others } \\
\text { Uncomfortable without close } \\
\text { relationships } \\
\text { Worry that others don't value them as } \\
\text { much as they value others }\end{array}$ \\
\hline Negative & $\begin{array}{l}\text { DISMISSING } \\
\text { Comfortable without close relationships } \\
\text { Import to feel independent and self- } \\
\text { sufficient } \\
\text { Prefer not to depend on others or have } \\
\text { others depend on them }\end{array}$ & $\begin{array}{l}\text { FEARFUL } \\
\text { Somewhat uncomfortable getting } \\
\text { close to others } \\
\text { Difficulty trusting others of depending } \\
\text { on them } \\
\text { Worry that will be hurt if get close to } \\
\text { others }\end{array}$ \\
\hline
\end{tabular}


Attachment security/insecurity is assessed in various ways. One popular way, particularly among clinicians is the Adult Attachment Interview (AAI; Main, Kaplan \& Cassidy, 1985) that looks at the coherence with which adults can report on their early attachment relationships. Another method of assessment is to use a categorical measure of attachment such as that devised in the classic study of Hazan and Shaver (1987) which was the study that initiated a large amount of research on adult attachment carried out in the 1980s, 1990s and later. This categorical measure involved providing participants with descriptions of three attachment styles: Secure, Avoidant and Anxious-ambivalent and having them choose which description was most applicable to them. Later, Bartholomew and Horowitz (1991) designed a measure suitable for assessing the four styles involved in their model discussed earlier: Secure, Preoccupied, Dismissing and Fearful.

Although much research has been stimulated by the availability of categorical measures, there are also problems with this type of assessment. For example, as we noted earlier, the four styles are not evenly distributed among members of the population. As reported earlier, Hazan and Shaver (1987) found that the distribution of attachment styles among adults was similar to the distribution among infants as found by Ainsworth et al., (1978) with about 50 percent or so reporting that they are secure and the rest being spread over the other categories. Thus using a categorical measure in research studies can be problematic, because of uneven cell sizes that make analysis difficult.

For this reason, continuous measures of attachment have been developed with the goal of providing measures of the dimensions central to attachment. The advantage of dimensional measures for researchers is that all participants obtain a score on each of the dimensions and there are no cell-size problems. In addition, participants do not have to choose between three or four categories that they may not see as particularly relevant to them. The Attachment Style Questionnaire (ASQ; Feeney, Noller \& Hanrahan, 1994), for example, assessed five dimensions: confidence in self and others, preoccupation, need for approval, discomfort with closeness and relationships as secondary. The Experiences in Close Relationships questionnaire (Brennan, Clark \& Shaver, 1998) focuses on two main dimensions: anxiety and avoidance that have been shown to clearly differentiate between the attachment styles.

\subsubsection{Transfer of Attachment}

Allen and Land (1999) discuss the similarities and differences between infant attachment and adolescent attachment. For example most young people in mid adolescence will seek comfort and help from their parents when they are highly distressed, even as they reach young adulthood. On the other hand, they have a strong need to break their dependency on their parents, and to develop strong relationships with peers, including romantic partners. Hence they do not relinquish attachment 
needs and behaviours, but rather transfer them to peers, particularly a romantic partner.

We know that the attachment functions discussed earlier (proximity seeking, separation protest, safe haven and secure base) are not all transferred to peers or a romantic partner at the same time (Hazan \& Zeifman, 1994), and the stage at which they are transferred can vary from one person to another. These researchers found that more than half of the children as young as 6 or 7 preferred to spend time with their peers rather than their parents (proximity seeking), more than $60 \%$ of the early adolescents (11-14) preferred to talk to their peers rather than their parents about their problems (safe haven) and that only about 40\% of 15-17 year olds had transferred the secure base function. It seems that even in their twenties, about $60 \%$ of young people preferred their parents as their secure base (Fraley \& Davis, 1997; Trinke \& Bartholomew, 1997). In general, the secure base function does not seem to be fully transferred until adulthood (Friedlmeier \& Granquist, 2006).

Transfer of attachment also depends on whether a person is in a romantic relationship (Hazan \& Zeifman, 1994). Being involved in a romantic relationship for two years increased the chances that the attachment functions were fully transferred from the parents to the romantic partner. For those who were not in a long-term relationship, friends were mainly used for the proximity and safe haven functions, and parents still provided the secure base. In addition, in the absence of a romantic partner, close friendships lasting more than five or so years can be full attachment relationships fulfilling all the attachment functions (Fraley \& Davis, 1997).

\subsection{Life Course Theory}

Life Course theory (Elder, 1998a, 1998b) focuses on both the social situation in which a person is developing and the individual's need to make decisions and act on their own behalf. The emphasis is on people's stories that unfold over time as they grow and mature and as society changes. Elder developed four principles, with the first principle focusing on the way that individuals' lives are shaped by the time and place in which they are born and grow up. For example, those individuals (like the first author) who were born soon after the Great Depression and who grew up during the Second World War will have had different experiences from those who were born in the boom times of the 50s and 60s. Elder (1999) found that these two traumatic historical events had a large impact on the American young people that he studied.

Elder's second principle was that it's not just the historical events that a person lives through that have an impact on them, but that the timing of the events is really important. For example, those young people in Elder's studies (1998a) who were born in 1920 and were adolescents during the Great Depression and the war seemed to fare better than those who were children during the Depression and adolescents during 
the war. This latter group tended to be plagued by feelings of personal and social inadequacy, probably because so much of their early life was lived in deprivation and fear.

Elder's third principle emphasized the interdependence of our lives that are entwined in a network of relationships in the family and beyond. For example, young people are likely to be affected by outside events that don't impinge on their lives directly but that affect them through their parents. For example, if the global financial crisis of 2008-9 caused their father to lose his job, they may have to leave school to ease the financial burden on the family and miss out on qualifying for university. If conflict increases in the home because of the financial situation, the adolescent's life is likely to become more difficult, particularly if he or she gets caught up in the parents' conflict. If the parents end up divorcing, he/she may have to move to a less affluent suburb and leave friends behind. (See Chapter 5 for more on adolescents and divorce in the family) How well an adolescent copes with all these changes is likely to depend on the levels of social support. If there is plenty of support from family and friends, adolescents are likely to cope better, as did the young rural sample that Elder studied following the Great Farm Crisis in the US in the 80s.

Elder's fourth principle focuses on the fact that, to a great extent, individuals make their own lives by the choices they make and by the extent to which they make good use of the opportunities that come their way, or waste those opportunities. The young person who wins a scholarship to university and then wastes time in social activities to the extent that exams are failed and a scholarship lost has made a poor life choice. Recovery from that bad choice depends on the next choice made. The young person, on the other hand, who grabs the opportunity that a scholarship provides and works hard is likely to be rewarded with good grades and a worthwhile career.

It is clear that the social context of the $21^{\text {st }}$ Century provides many choices for young people, some that will make their lives easier and some that will make their lives harder. It is important to remember, however, that historical conditions, the social environment (family, school, work etc.) and the characteristics of the young person (both strengths and weaknesses) all work together to produce the person (Elder \& Conger, 2000).

\subsection{Positive Youth Development}

Stemming to some extent from the work of Elder (1994) as well as from the Positive Psychology movement, there has been a call more recently for adolescent development to be viewed through a more positive lens. Positive Youth Development (PYD) is a framework that has been introduced to further this aim (Hamilton, Hamilton \& Pittman (2004). This framework is strengths-based and acknowledges that adolescents are instrumental in their own development, whilst being influenced by their personal, family and environmental resources (Benson, Scales \& Mannes, 
2003; Damon, 2004; Lerner, Brentano, Dowling, \& Anderson, 2003). This model is not yet well researched or formulated but it does have some obvious usefulness (Klatt \& Enright, 2009; Larson 2000; 2006).

In discussing Positive Youth Development (PYD) here, we focus on PYD as a philosophy characterized by an orientation toward building upon strengths and assets, rather than focusing on deficits in young people. According to Small and Memmo (2004), PYD has four key assumptions as its foundation. The assumptions include (1) adolescents can best be prevented from developing problems when they are assisted to achieve their full potential; (2) adolescents are more likely to succeed when they are supported and have appropriate opportunities; (3) communities need to be involved with their youth and invest in their futures; (4) there is a need to change community attitudes towards young people so that they are no longer seen as "problems to be fixed" but as partners capable of self-determination.

As do resilience models (e.g. Masten, 2001), PYD emphasises the importance of stability, care, healthy relationships and high expectations, in terms of having confidence in young people and providing opportunities for them. The Search Institute model of PYD has been instrumental in the development and promotion of PYD (Bradshaw, Brown \& Hamilton, 2006; Small \& Memmo, 2004). This model is based around 40 developmental assets that are categorised into 2 broad areas; internal factors (commitment to learning, positive identity and social competence) and external factors (support, empowerment, boundaries and expectations and constructive use of time). The aim of PYD is to facilitate the positive development of young people's assets and asserts that the more assets young people develop the more likely they are to engage in prosocial behaviour and the less likely they are to engage in problematic behaviour (Scales, Benson, Leffert \& Blyth, 2000).

Larson (2000) perceives PYD as a useful model for understanding how we can assist young people to develop initiative (independent action), which is an important attribute in western culture and one that adolescents need to develop. This process can be aided by activities that challenge young people and give them skills of thinking, planning and achievement that can be carried into other areas of their lives.

There has been a proliferation of programs for adolescents using this model, particularly in the United States (Bradshaw, Brown \& Hamilton, 2008), even with juveniles involved with the justice system. Bradshaw et al. claim “The appeal of these programs appears to stem from their focus on youths' assets rather than their deficits, and a positive rather than a problem-centred approach” (p. 209).

One of the positive aspects of the PYD model is that, because it promotes strengths and assets, it can be applied to all youth and not just to well-behaved youth or to young people labelled as problems (Small \& Memmo, 2004). Small and Memmo also point out that the approach fits with the emphasis on "political correctness" that, from our perspective, can often limit our ability to see the world as it really is and to explore ways to address problems directly. As Small and Memmo also note, however, an asset-based approach can be very helpful to those who work with young people. 
On the other hand, we are concerned that an over-reliance on the PYD approach may downplay the importance of understanding the risks young people can face and the poor choices they may make. Yet an understanding of such issues is critical to being able to provide help and support when needed. It is quite clear that some adolescents, even though in the minority, develop problematic behaviour that can lead to even more problems in adulthood (Bradshaw et al.,2006; Piquero, Farrington \& Blumstein, 2003). In these instances, we believe that a combination of the strengths approach of PYD and more clinical interventions to address the problematic behaviour is required; this position is supported by Bradshaw et al. and Small and Memmo (2004).

\subsection{Summary}

Although adolescence used to be seen as a time of 'storm and stress' for all adolescents, we now understand that most adolescents are able to negotiate this part of their lives without too much trauma. Most adolescents do not have severe mood swings, are able to maintain relatively harmonious relationships with their parents and to make constructive life choices.

Nevertheless, adolescence is an egocentric time, with adolescents generally being preoccupied with themselves and their own issues, and also being very selfconscious. They tend to see themselves as surrounded by an imaginary audience that is always watching them and tend to conform to the personal fable that they are special and unique and invulnerable to harm.

Two processes that adolescents need to go through in order to become mature adults are separation-individuation and identity formation. Separation-individuation involves separating from the family and becoming an autonomous person who is able to make decisions and lead an independent life. Ideally young people can develop a sense of self that is separate from family and still be able to maintain connections to them. It is interesting to note that those who individuate successfully tend to develop a greater capacity for close relationships.

The second process they need to go through is identity formation. They need to explore alternatives and decide who they are and what they want to become. Ideally, parents will encourage them to think, feel and act independently of family members, making the development of a stable identity easier. A stable identity will emerge from choices about vocation, political preferences, religious commitments and sexual orientation and many others. As adolescents receive feedback about their identity choices, they may choose to modify their identity or ignore such feedback.

Secure attachment relationships perform four functions: proximity seeking, separation protest, secure base and safe haven. Secure adolescents tend to have more positive relationships with their parents than do insecure adolescents and are better able to achieve autonomy and independence in the context of secure relationships with their parents. 
Attachment functions tend to be transferred from parents to peers and later to a romantic partner, although the secure base function will be the last to be transferred. Being in a romantic relationship for two years increases the chances that all four attachment functions will be transferred to the partner.

Elder developed four principles about growth and maturity across the life course. The first principle is about how individuals' lives are shaped by the time and place in which they are born and grow up. The second principle focuses on the timing of particular events in a person's life as also important, because the effects might differ depending on life stage. The third principle emphasized the interdependence we all have with family and friends and how factors that affect the parents, for example, will also affect the adolescent offspring. The fourth principle focuses on individual responsibility for making choices and how lives can be affected for good or for ill by good or poor choices.

The work of Elder has been influential in the formulation of Positive Youth Development, a framework for encouraging the viewing of adolescents through a more positive lens. This approach focuses on the strengths and assets of adolescents rather than their problem behaviours and has spawned a range of programs for helping adolescents, even those with serious problems, to become more competent and motivated.

As previously mentioned, most adolescents learn to feel and act independently of family members within the context of harmonious and secure relationships with their parents. A crucial aspect of family relationships as adolescents develop is the quality of the communication between family members. In the next chapter we look at the importance of the family environment to the well-being of adolescents. 